\title{
El tratamiento con implantes dentales postextracción
}

\author{
VELASCO ORTEGA E * \\ PATO MOURELO J ** \\ LORRIO CASTRO JM *** \\ CRUZ VALIÑO JM** \\ POYATO FERRERA $\mathbf{M} * * * *$
}

\begin{abstract}
Velasco Ortega E, Pato Mourelo J, Lorrio Castro JM, Cruz Valiño JM, Poyato Ferrera M. El tratamiento con implantes dentales postextracción. Av Periodon Implantol. 2007; 19, Suppl: 35-42.
\end{abstract}

\begin{abstract}
RESUMEN
Introducción. El objetivo del presente estudio era mostrar los resultados de del tratamiento con implantes dentales insertados inmediatamente después de la extracción.

Métodos. 22 pacientes con pérdida dental unitaria, parcial o total fueron tratados con 82 implantes Microdent ${ }^{\circledR}$ con superficie con chorreado de arena y grabada con ácidos. Todos los implantes fueron insertados inmediatamente después de la extracción correspondiente. Los implantes fueron cargados después de un periodo de cicatrización de 3 meses en la mandíbula o 6 meses en el maxilar superior.
\end{abstract}

Resultados. Los hallazgos clínicos indican una supervivencia y éxito de los implantes del $97,6 \%$. 2 implantes se perdieron durante el periodo de cicatrización. El 73,2\% de los implantes fueron insertados en el maxilar. superior mientras que el $26,8 \%$ en la mandíbula. Después de un periodo medio de carga funcional de 12 meses, no ha habido complicaciones tardías.

Conclusiones. Este estudio indica que los implantes dentales insertados de forma inmediata después de la extracción pueden constituir una alternativa implantológica predecible y exitosa.

\section{PALABRAS CLAVE}

Implantes orales, implantes postextracción, biomateriales, implantología oral.

Fecha de recepción: Diciembre 2006.

Fecha de aceptación: Enero 2007.

\section{INTRODUCCIÓN}

La implantología oral ha revolucionado la práctica odontológica cotidiana al incorporar una técnica terapéutica predecible para la rehabilitación oral de los pacientes que por diferentes causas han perdido sus dientes naturales. En este sentido, numerosos pacientes han sido tratados mediante la rehabilitación protésica con implantes oseointegrados con unos resultados funcionales y estéticos excelentes ${ }^{1}$.

* Profesor Titular de Odontología Integrada de Adultos. Facultad de Odontología. Director del Postgrado de Implantología Oral. Universidad de Sevilla.

** Profesor Colaborador Honorario de Odontología Integrada de Adultos. Facultad de Odontología. Universidad de Sevilla.

*** Profesor Residente de Odontología Integrada de Pacientes Especiales. Facultad Odontología. Universidad Alfonso X el Sabio. Madrid.

**** Profesor Asociado de Odontología Preventiva y Comunitaria. Facultad de Odontología. Universidad de Sevilla. 
La evolución de la implantología oral mediante la investigación experimental y clínica ha hecho posible el desarrollo de técnicas quirúrgicas y prostodóncicas que han mejorado las expectativas de éxito entre los pacientes. En este sentido, se ha incorporado como técnica quirúrgica implantológica la inserción de implantes de forma inmediata tras la extracción del diente correspondiente sin posibilidad de tratamiento conservador ${ }^{2}$.

Hace más de 25 años que Schulte ${ }^{3}$ describió los primeros resultados de esta técnica con pacientes mediante la inserción de implantes Frialit después de un seguimiento clínico de 8 años. Block y Kent ${ }^{4}$ presentaron unos resultados con un éxito del $96,8 \%$ en un estudio a lo largo de 2 años realizado con 62 implantes recubiertos de hidroxiapatita insertados postextracción, utilizando como material de relleno en los defectos periimplantarios pequeños, hidroxiapatita y en los defectos mayores, hueso mineralizado.

Krump y Barnett ${ }^{5}$ presentan los resultados de la inserción de 41 implantes sumergidos inmediatamente después de la extracción en 11 pacientes comparados con 154 implantes sumergidos insertados de forma diferida en 35 pacientes sin diferencias significativas entre ambos grupos y un $93 \%$ de éxito en los insertados postextracción con un periodo de seguimiento de 1948 meses.

Gómez-Román y cols ${ }^{6}$ comparan los hallazgos clínicos de la inserción de 86 implantes postextracción Frialit y de 446 implantes Frialit insertados de forma diferida después de 9 meses de la extracción dental. Mientras que entre los implantes inmediatos la tasa de fracasos fue del 1,16\%; entre los implantes diferidos la tasa fue del $3,81 \%$.

Lang y cols ${ }^{7}$ recomiendan esta técnica al describir la utilización de 21 implantes no sumergidos ITI insertados de forma inmediata tras la extracción con regeneración tisular guiada mediante membranas en 16 pacientes. Sólo en un implante no hubo un relleno completo óseo que cubriera la superficie del implante.

Fugazzotto ${ }^{8}$ presenta los resultados de esta técnica con 162 implantes postextracción controlados durante 7 años, donde solo un implante presentó movilidad y fue extraído, lo que indica un éxito del 99,4\%. Este estudio incide sobre la importancia de realizar mediante un fresado una osteotomía apical en el alveolo independientemente de su morfología para conseguir la mejor estabilidad primaria del implante.
Más recientemente, dos estudios confirman la posibilidad de realizar esta técnica de inserción inmediata postextracción con un protocolo de carga inmediata funcional ${ }^{9-10}$. Cooper y cols. ${ }^{9}$ realizaron a 10 pacientes en una misma sesión, 54 implantes postextracción y carga inmediata en 48 de ellos con una prótesis fija de resina en la zona anterior mandibular. A las 12 semanas se le realizó la prótesis fija definitiva. Después de un periodo de seguimiento clínico entre 618 meses, no hubo ningún fracaso implantológico. Aires y Berger ${ }^{10}$ realizaron un estudio con 75 implantes en 7 pacientes, de los que 29 implantes fueron insertados en alveolos postextracción. Los 29 implantes postextracción fueron cargados de forma inmediata y 33 implantes de los demás también. 2 implantes fracasaron, uno de los implantes postextracción y otro en un lugar que no era de extracción, lo que indica que la tasa de éxito para los implantes cargados inmediatamente no difería si habían sido insertados de forma diferida o inmediata después de la extracción ${ }^{10}$.

El objetivo del presente estudio era la valoración clínica y el seguimiento del tratamiento con implantes oseointegrados mediante la técnica de inserción inmediata tras la extracción.

\section{PACIENTES Y IMETODOS}

El presente estudio fue realizado por profesores de las unidades docentes de Odontología Integrada de Adultos de la Facultad de Odontología y del Postgrado de Implantología Oral de la Universidad de Sevilla.

Previamente a la realización del estudio, aquellos pacientes que padecían trastornos sistémicos graves que podían comprometer la oseointegración fueron excluidos del estudio ${ }^{11}$. Los pacientes seleccionados eran adultos, de ambos sexos.

Todos los pacientes seleccionados en el estudio fueron informados de la técnica quirúrgica de inserción de implantes inmediatamente después de la extracción dental, así como del protocolo posterior del tratamiento incluyendo los aspectos prostodóncicos, temporalización y seguimiento, y de la posibilidad de la existencia de complicaciones y pérdida de implantes.

Los pacientes autorizaron el tratamiento implantológico mediante un consentimiento informado. Antes del tratamiento, todos los pacientes fueron evaluados radiológicamente, con una ortopantomografía. 
Los criterios de éxito y supervivencia de los implantes fueron los recomendados por van Steenberghe et $\mathrm{al}^{12}$. En este sentido, la supervivencia fue definida como la proporción de implantes permanentes en su localización original aunque no tengan valor clínico o cause efectos adversos. Los criterios de éxito de los implantes se expresan en la Tabla 1.

\section{Cirugía}

Una hora antes de la cirugía, los pacientes comenzaron un régimen antibiótico preventivo (amoxicilina + clavulánico) durante una semana. En casos de molestias, dolor o inflamación a todos los pacientes se les recomendó ibuprofeno. Todos los pacientes recibieron anestesia local.

Se realizó la técnica de inserción inmediata de los implantes después de la extracción dental correspondiente que esencialmente consiste en la realización de una exodoncia lo más atraumática posible y limpieza del alveolo con una cucharilla. A continuación se realiza un fresado apical a baja velocidad (800 r.p.m) profundizando aproximadamente $3 \mathrm{~mm}$ para conseguir la inserción del implante con una buena estabilidad primaria.

El espacio entre el implante y las paredes del alveolo no se rellenó con ningún biomaterial. A todos los pa-

\section{TABLA I. - LOS CRITERIOS DE ÉXITO EN EL TRATAIMIENTO CON IIMPLANTES (van Steenberghe et al ${ }^{12}$ )}

1. El implante no provoca ninguna reacción alérgica, tóxica o infeccciosa de carácter local o sistémico.

2. El implante ofrece soporte para una prótesis funcional.

3. El implante no muestra signos de fractura o incurvación.

4. El implante no muestra ninguna movilidad cuando es explorado manual o electrónicamente.

5. El implante no muestra ningún signo de radiolucidez mediante una radiografía intraoral.

6. La pérdida marginal de hueso ( $\mathrm{Rx}$ intraoral) y/o la pérdida de inserción (profundidad de sondaje + recesión) no deben perjudicar la función de anclaje del implante o causar molestias para el paciente durante 20 años. cientes se les recomendó el enjuague diario con clorhexidina durante los primeros 30 días.

Los implantes insertados fueron Microdent ${ }^{\circledR}$ (Microdent, Barcelona, España) roscados de superficie con chorreado de arena y grabado ácido, y todos fueron estables después de la inserción.

\section{Prostodoncia}

A los 3-6 meses, en maxilar superior o mandíbula, respectivamente, se realizó la carga funcional de los implantes mediante la colocación de las correspondientes prótesis implantosoportadas. El tiempo transcurrido de seguimiento clínico desde la carga funcional de los implantes fue al menos de 12 meses.

\section{ANÁLISIS ESTADÍSTICO}

Se realizó una estadística descriptiva de los hallazgos clínicos del estudio, con referencia a las variables demográficas de los pacientes, la inserción, supervivencia y pérdida de los implantes así como de las restauraciones prostodóncicas realizadas.

\section{RESULTADOS}

\section{Pacientes}

El presente estudio incluía a 22 pacientes con pérdidas dentales unitarias, parciales y totales, de los cua-

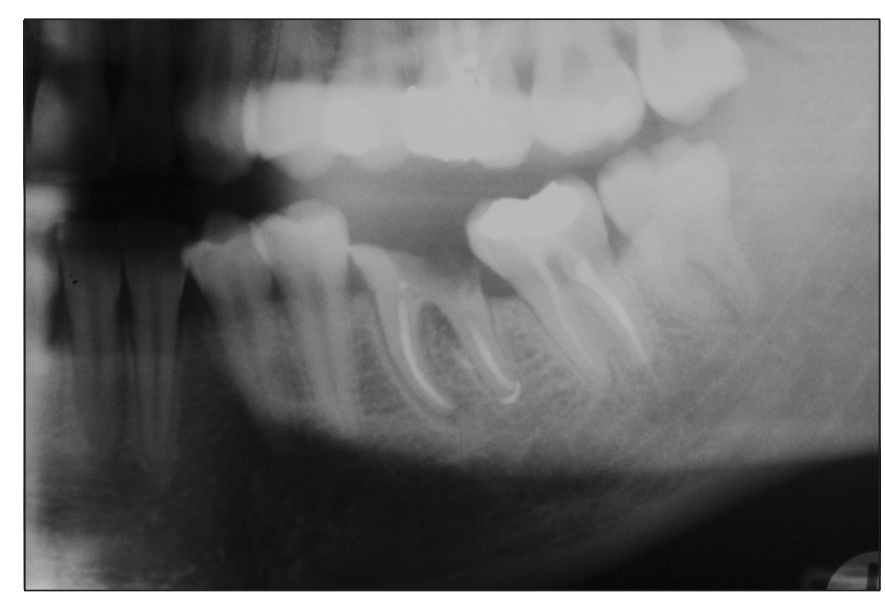

Fig. 1: Diagnóstico radiológico del paciente. Resto radicular en el 36 . 


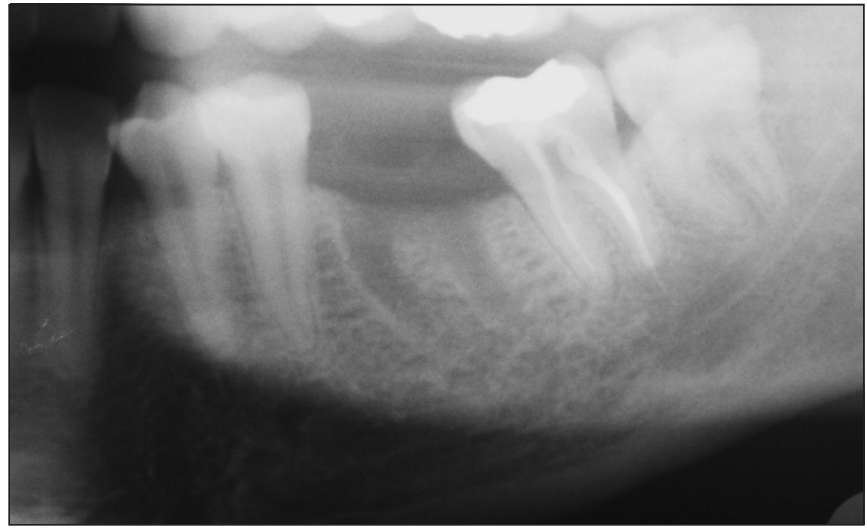

Fig. 2: Diagnóstico radiológico del paciente. Resto radicular extraído.

les 9 eran hombres y 13 eran mujeres con una edad media de 49,8 años (rango: 39-62 años). 11 pacientes $(50 \%)$ tenían antecedentes periodontales. 4 pacientes eran fumadores $(18,2 \%)$.

\section{IMPLANTES}

Se insertaron un total de 82 implantes Microdent en los correspondientes 22 pacientes, lo que representa una media de 3,7 implantes por paciente.

Con respecto al diámetro se insertaron 4 implantes de $3,3 \mathrm{~mm}(4,9 \%), 5$ implantes de 3,8 mm $(6,1 \%)$ y $73 \mathrm{im}-$ plantes de $4,2 \mathrm{~mm}(89 \%)$. Con respecto a la longitud, fueron insertados 81 implantes de $12 \mathrm{~mm}(98,8 \%)$ y 1 implante de $14 \mathrm{~mm}(1,2 \%)$.

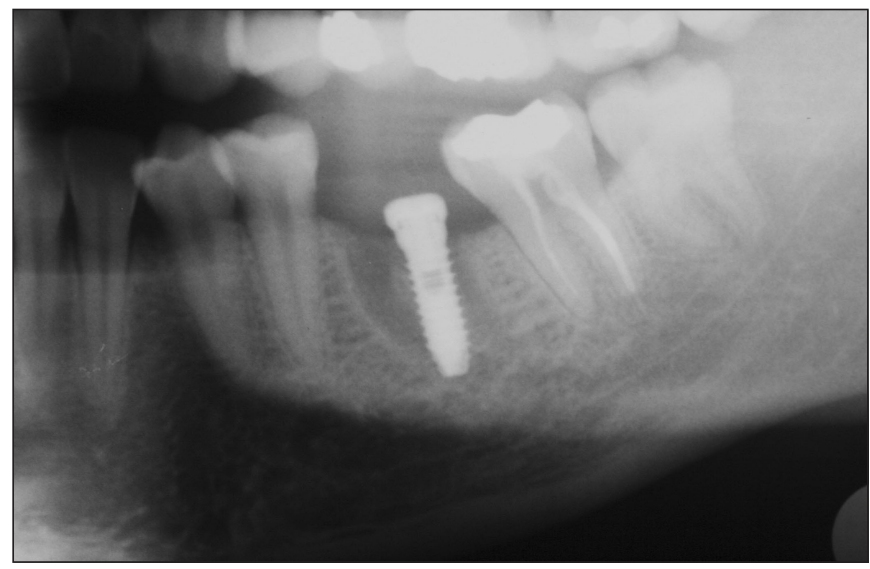

Fig. 3: Diagnóstico radiológico del paciente. Implante de conexión externa 4/12 Microdent insertado inmediatamente después de la extracción.
En todos los pacientes los implantes se insertaron de forma inmediata en el mismo acto quirúrgico de la extracción dental en el maxilar superior y en la mandíbula. 60 implantes $(73,2 \%)$ fueron insertados en el maxilar superior, 30 implantes en el sector anterior (incisivos y caninos) y 30 implantes en el sector posterior (premolares y molares). 22 implantes $(26,8 \%)$ fueron insertados en la mandíbula, 18 implantes en el sector anterior (incisivos y caninos) y 4 implantes en el sector posterior (premolares y molares).

En 2 implantes $(2,4 \%)$ hubo complicaciones, al presentar movilidad a la exploración clínica durante la fase de cicatrización libre de carga funcional y fueron extraídos. Ningún implante se ha perdido después de la carga funcional prostodóncica durante el periodo de seguimiento clínico.

\section{Prótesis IMPLANTOSOPORTADAS}

Los 80 implantes restantes fueron cargados funcionalmente, a los 3 meses en la mandíbula y a los 6 meses en el maxilar superior. Se realizaron 27 prótesis sobre implantes, 11 coronas unitarias $(40,8 \%), 8$ puentes o prótesis fijas $(29,6 \%), 4$ rehabilitaciones totales fijas $(14,8 \%)$ y 4 sobredentaduras $(14,8 \%)$. El seguimiento clínico medio ha sido de 12 meses.

\section{DISCUSIÓN}

Este estudio valora los resultados clínicos del tratamiento con implantes oseointegrados mediante la técnica de inserción inmediata después de la extracción del diente correspondiente. Esta técnica quirúrgica introducida por Schulte ${ }^{3}$ en 1978 se ha ido incorporando progresivamente a la práctica implantológica general con buenos resultados ${ }^{13-15}$.

Los fenómenos biológicos que ocurren después de la extracción dental han sido estudiados en estudios con animales de experimentación y en biopsias con pacientes ${ }^{16-17}$. Los diferentes estadios que configuran la cicatrización del alveolo y la progresiva sustitución por tejido óseo duran entre 4-6 semanas, aunque la remodelación definitiva puede durar hasta 4 meses ${ }^{18-19}$.

Después de la extracción ocurren cambios morfológicos importantes. Aproximadamente entre 5-7 mm se reduce la distancia horizontal o anchura vestíbulo lingual después un periodo de 6-12 meses después de la extracción, lo que representa casi el $50 \%$ de la an- 


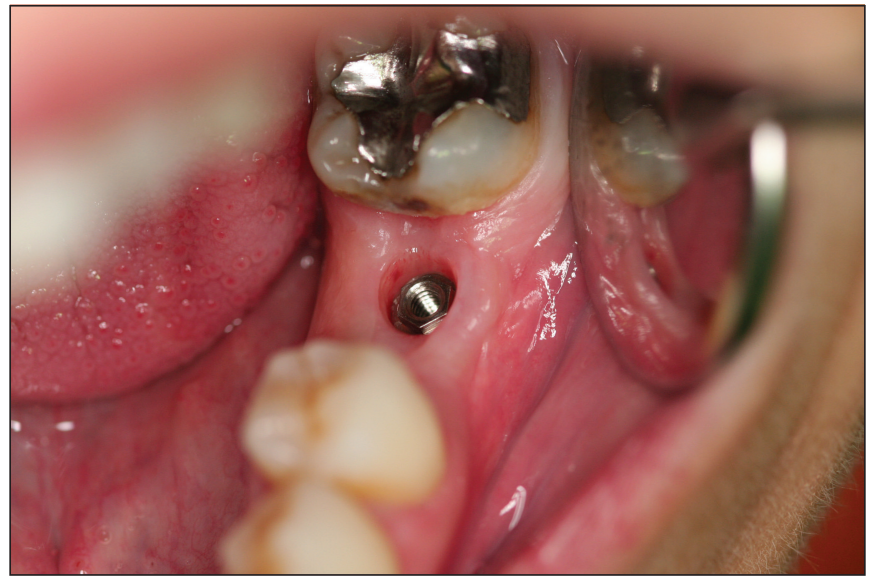

Fig. 4: Aspecto clínico a los 3 meses.

chura alveolar inicial. La mayoría de estos cambios tienen lugar en los 4 primeros meses de cicatrización. A estos cambios horizontales se acompañan cambios en la altura o apicoronales con una reducción de 2 a 4,5 $\mathrm{mm}$. Sobre todo si son varias las extracciones realiza$\operatorname{das}^{20-21}$.

Las ventajas de esta técnica incluyen una reducción importante en el número de intervenciones quirúrgicas y en el tiempo de tratamiento. También se puede utilizar el alveolo como guía de la orientación del implante. Otros ventajas son la preservación del hueso alrededor de la extracción y la buena respuesta de los tejidos blandos que mejoran la estética final ${ }^{22-24}$. En

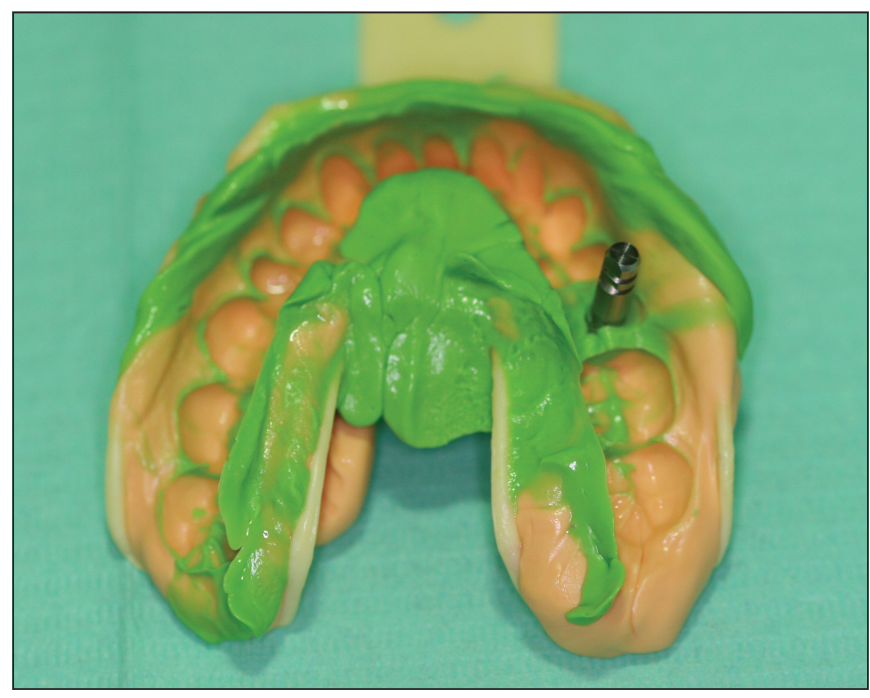

Fig. 5: Toma de impresión con análogo de implante colocado.

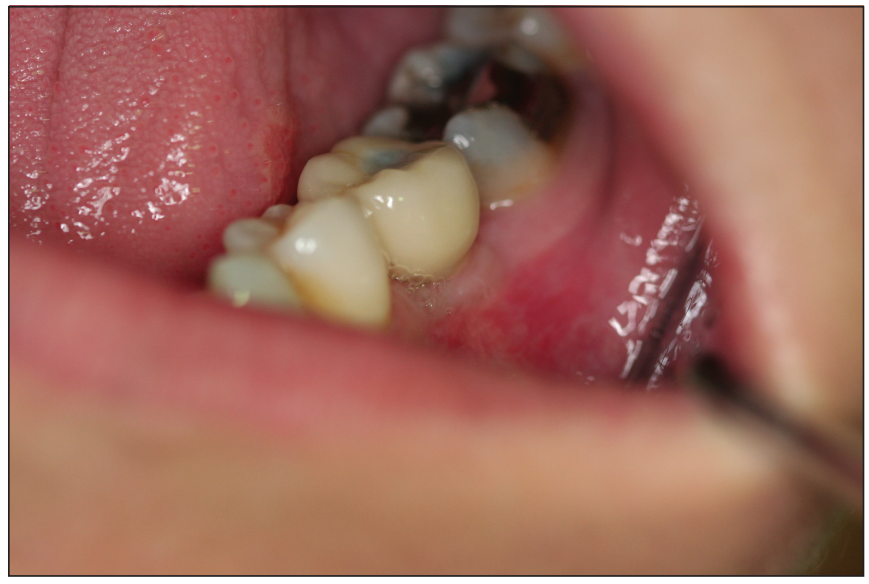

Fig. 6: Aspecto clínico de la corona implantosoportada.

este sentido, la inserción de implantes de forma inmediata después de la extracción puede favorecer una mejor cicatrización del alveolo y prevenir los cambios morfológicos derivados de la cicatrización y remodelación ósea ${ }^{13}$.

Sin embargo esta técnica de inserción inmediata de implantes postextracción puede presentar complicaciones cuando se utiliza en zonas con presencia de infección o lesiones periapicales quísticas. Así mismo, la repuesta tisular ósea y mucosa periimplantaria puede afectarse cuando existe una gran discrepancia entre el tamaño del alveolo y el del implante que pueda originar un colapso del volumen tisular o una exposición de la membrana, en los casos de regeneración tisular guiada ${ }^{25-27}$.

Aunque, en el presente estudio no se han realizado técnicas de regeneración tisular; sin embargo, se ha recomendado la utilización de material de relleno o biomateriales con membranas para obtener una mejor cicatrización ósea y reducir la pérdida ósea crestal comparados con otras localizaciones donde se utilizaron solamente las membranas ${ }^{28}$. De hecho, esta técnica de regeneración ósea puede ser recomendada sobre todo cuando se han producido dehiscencias o pérdida de alguna pared ósea durante la extracción que pudiera comprometer la inserción inmediata del implante ${ }^{29}$.

Un estudio realizado con 96 miniimplantes experimentales ITI en 48 pacientes demostró que no había diferencias en la cicatrización ósea entre las localizaciones postextracción y las situadas en hueso maduro a los 6 meses de la inserción de los implantes. En el estudio no se realizó ni regeneración tisular guiada ni injertos ${ }^{30}$. 
Aunque no es imprescindible, la técnica de aumento del volumen óseo por regeneración guiada es recomendable cuando existe una distancia mayor de $2 \mathrm{~mm}$ entre la superficie del implante y la pared del alveolo ${ }^{31}$. Ahora bien, cuando se utilizan membranas, existe el riesgo de su temprana exposición y se reduce entonces, la calidad y cantidad del hueso neoformado en los defectos periimplantarios ${ }^{26,28}$.

Los resultados del presente estudio demuestran una elevada tasa de éxito $(97,6 \%)$ de los implantes insertados inmediatamente después de la extracción dental correspondiente durante un seguimiento clínico de 12 meses. Estos hallazgos clínicos se sitúan entre las tasas de supervivencia (89,9\% al $100 \%)$ de una revisión de 18 estudios de implantes postextracción seguidos entre 6 y 67 meses $^{13}$.

El diseño macroscópico de los implantes es importante para obtener una buena estabilidad primaria durante la fase quirúrgica como paso previo en la inserción inmediata postextracción. El presente estudio demuestra que los resultados obtenidos con los implantes Microdent insertados de forma inmediata postextracción son similares a los obtenidos con otros sistemas de implantes (Branemark, Calcitek, Frialit, IMZ, Integral, ITI, Steri-Oss) en las mismas condiciones clínicas ${ }^{13}$.

El diseño microscópico de los implantes, especialmente relacionado con la superficie rugosa es también importante porque se ha demostrado que favorece la oseointegración ${ }^{31-33}$. El presente estudio demuestra que con implantes con una superficie rugosa con chorreado de arena y grabado ácido (Microdent) se pueden obtener similares resultados de éxito en la inserción inmediata postextracción $(97,4 \%)$ a los implantes con hidroxiapatita (96-100\%), plasma de titanio $(100 \%)$, chorreado y grabado $(97 \%)$ y otras superficies $(89,3-99,4 \%)^{13}$.

Después de la carga funcional no se ha perdido ningún implante postextracción, habiéndose realizado diversas soluciones prostodóncicas, coronas unitarias $(40,8 \%)$, prótesis fija parcial $(29,6 \%)$, rehabilitaciones fijas implantosoportadas $(14,8 \%)$ y sobredentaduras $(14,8 \%)$.

Diversos estudios han demostrado que independientemente de la carga funcional realizada (convencional, precoz e inmediata), la respuesta ósea tras la inserción inmediata de implantes después de la extracción correspondiente es favorable y los resultados clínicos son muy buenos ${ }^{9-10}$. En este sentido, cuando se realiza una buena selección del paciente, se puede acortar notablemente el tiempo y las visitas clínicas al paciente, realizando de forma simultánea las extracciones indicadas, la inserción inmediata de los implantes y la restauración inmediata con una prótesis provisional de resina ${ }^{9}$.

Finalmente, un estudio muy reciente demuestra que la inserción de implantes postextracción favorece la estética posterior ya que preserva los tejidos periimplantarios, tanto mucosos como los niveles crestales óseos, aunque a veces es necesario la utilización de biomateriales o membranas ${ }^{34}$.

\section{CONCLUSIONES}

El tratamiento con implantes oseointegrados insertados de forma inmediata después de la extracción puede constituir una alternativa implantológica con un elevado éxito. La realización de una técnica quirúrgica cuidadosa que incluye la exodoncia menos traumática posible, la inserción más apical del implante con una buena estabilidad primaria, utilizando a veces la regeneración tisular guiada y biomateriales, permite la realización de este tratamiento e incluso la carga funcional inmediata, mejorando la calidad del tratamiento desde el punto de vista del paciente y previniendo las reducciones morfológicas del reborde alveolar edéntulo.

\section{ABSTRACT}

Introduction. The aim of this study was to report the outcome of treatment with implant inserted immediately after tooth extraction.

Methods. 22 patients with single, partially or totally tooth loss were treated with 82 Microdent ${ }^{\circledR}$ sandblastedetched surface implants. All implants were inserted immediately after tooth extraction. Implants were loaded after a healing free-loading period of 3 months in mandible or 6 months in maxilla.

Results. Clinical results indicate a survival and success rate of implants of 97,6\%. 2 implants were lost during the healing period. $73.2 \%$ of implants were inserted at maxilla while $26.8 \%$ were inserted at mandible. After a functioning period of 12 months, no late complications were reported.

Conclusions. This study indicate that dental implants inserted immediately after tooth extraction may 
constitute a successful, predictible alternative implant treatment.

\section{KEY WORDS}

Oral implants, postextraction implants, biomaterials, implant dentistry.

\section{BIBLIOGRAFIA}

1. Misch CE. Contemporary Implant Dentistry. $2^{\mathrm{a}}$ ed. San Luís: Mosby. 1999.

2. Peñarrocha M, Sanchís JM. Implante inmediato a la extracción. En: Peñarrocha M. Implantología oral. Barcelona: Ars Médica. 2001; pag: 85-93.

3. Schulte W, Kleineikenscheidt H, Linder K, Schareyka R. The Tubingen immediate implant in clinical studies. Dtsch Zahnärztl Zeitschr 1978; 33: 348-59.

4. Block MS, Kent JN. Placement of endosseous implants into tooth extraction sites. J Oral MaxilloFac Surg 1991; 49:1269-1276.

5. Krump JL, Barnett BG. The immediate implant: a treatment alternative. Int J Oral Maxillofac Implant 1991; 6:19-23.

6 Gómez-Román G, SchulteW, d'Hoedt B, Axman-Krcmar D. The Frialit-2 implant system: five-year clinical experience in single-tooth and immediately postextraction applications. Int J Oral Maxillofac Implant 1997;12:299-309.

7. Lang NP, Brägger U, Hämmerle CHF, Sutter F. Immediate transmucosal implant using the principle of guided tissue regeneration. Clin Oral Impl Res 1994;5:154-163.

8. Fugazzotto PA. Simplified technique for immediate implant insertion into extraction sockets: report of technique and preliminary results. Impl Dent 2002;11: 79-82.

9. Cooper LF, Rahman A, Moriarty J, Chaffee N, Sacco D. Immediate mandibular rehabilitation with endosseous implants: simultaneous extraction, implant placement, and loading. Int J Oral Maxillofac Implant 2002; 17:517-25.

10. Aires I, Berger J. Immediate placement in extraction sites followed by immediate loading: a pilot study and case presentation. Impl Dent 2002;1 1:87-94.
11. Buser D, von Arx T, ten Bruggenkate C, Weingart D. Basic surgical principles with ITI implants. Clin Oral Impl Res 2000;1 1(suppl 1):59-68.

12. van Steenberghe D, Quirynen, Naert I. Survival and success rates with oral endosseous implants. En: Lang NP, Karring T, Lindhe J (eds.). Proceedings of the 3rd European Workshop on Periodontology. Implant Dentistry. Berlin: Quintessence. 1999. pag: 242-52.

13. Chen ST, Wilson TG, Hämmerle CHF. Immediate or early placement of implants following tooth extraction: review of biologic basis, clinical procedures and outcomes. Int J Oral Maxillofac Implants 2004; 19 (suppl): 12-25.

14. Schwartz-Arad D, Chaushu G. The ways and werefores of immediate placement of implants into fresh extraction sites: a literature review.J Periodontol 1997;68: 915-23.

15. Mayfield LJA. Immediate, delayed and late submerged and transmucosal implants. En: Lang NP, Karring T, Lindhe J (eds.). Proceedings of the 3rd European Workshop on Periodontology. Implant Dentistry. Berlin: Quintessence. 1999. pag: 520-34.

16. Huebsch RF, Hansen LS. A histopathologic study of extraction wound in dogs. Oral Surg Oral Med Oral Pathol 1969;272:187-96.

17. Boyne PJ. Osseus repair of the postextraction alveolus in man. Oral Surg Oral Med Oral Pathol 1966; 21 : 805-13.

18. Amler MH. The time sequence of tissue regeneration in human extraction wounds. Oral Surg Oral Med Oral Pathol 1969;273:309-18.

19. Evian CI, Rosenberg ES, Cosslet JG, Corn H. The osteogenic activity of bone removed from healing extraction sockets in human. J Periodontol 1982;53:81-5.

20. Johnson K. A study of the dimensional changes occurring in the maxilla following tooth extraction. Aust Dent J 1969;14:241-4.

21. Lam RV. Contour changes of the alveolar processes following extractions. J Prosthet Dent 1960;10:25-32.

22. Lazzara RM. Immediate implant placement into extraction sites: surgical and restorative advantages. Int J Periodontics Restorative Dent 1989;9:333-43. 
23. Werbitt MJ, Goldberg PV. The immediate implant: bone preservation and bone regeneration. Int J Periodontics Restorative Dent 1992;12:207-17.

24. Denissen HW, Kalk W, Veldhuis HA, van Waas MA. Anatomic considerations for preventive implantation. Int J Oral Maxillofac Implants 1993;8:191-196.

25. Rosenquist B, Grenthe B. Immediate placement of implants into extraction sockets: implant survival. Int J Oral Maxillofac Implants 1996;1 1:205-9.

26. Augthun M, Yildrim M, Spiekermann H, Biesterfeld S. Healing of bone defects in combination with immediate implants using the membrane technique. Int J Oral Maxillofac Implants 1995;10:191-6.

27. Block MS, Kent JN. Factors associated with soft and hard tissue compromise of endosseous implants. J Oral Maxillofac Surg 1990;5:1153-60.

28. Gher ME, Quintero G, Assad D, Monaco E, Richardson AC. Bone grafting and guided bone regeneration for inmediate dental implants in humans. J Periodontol 1994; 65:881-91.

29. Zitzmann NU, Schärer P, Marinello CP. Factors influencing the success of GBR. Smoking, timing of implant placement, implant location, bone qualityu and provisional restoration. J Clin Periodontal 1999;26:673-82.
30. Paolantonio M, Dolci M, Scarano A et al. Immediate implantation in fresh extractions sockets. A controlled clinical and histological study in man. J Periodontol 2001;72:1560-71.

31. Covani U, Cronelini R, Barone. Bucco-lingual bone remodelling around implants placed into immediate extraction sockets. A case series. J Periodontol 2003;74: 268-73.

32. Jokstad A, Braegger U, Brunski JB, Carr AB, Naert I, Wennerberg $A$. Quality of dental implants. Int J Prosthodont 2004;17:607-41.

33. Ellingsen JE, Thomsen P, Lynstadaas P. Advances in dental implant materials and tissue regeneration. Periodontology 2000 2006;41:136-56.

34. Juodzbalys G,Wang HL. Soft and hard tissue assessment of immediate implant placement: a case series. Clin Oral Impl Res 2007. En prensa.

\section{CORRESPONDENCIA}

Prof. Eugenio Velasco Ortega

Facultad de Odontología de Sevilla

Avicena, s/n

41009 Sevilla

e-mail: evelasco@us.es 